\title{
Optical tracking filter using transient energy coupling
}

\author{
Norman Sze-Keung Kwong \\ Ortel Corporation, 2015 West Chestnut Street, Alhambra, California 91803
}

Yasuo Tamita and Amnon Yariv

Department of Applied Physics, 128-95, California Institute of Technology, Pasadena, California 91125

Received January 4, 1988; accepted March 23, 1988

\begin{abstract}
Transient energy coupling between two coherent beams occurs in dynamic holographic media with local and noninstantaneous responses. The physical origin of the effect is described, and an optical tracking filter based on the effect is demonstrated in a photorefractive $\mathrm{Bi}_{12} \mathrm{SiO}_{20}$ crystal.
\end{abstract}

\section{INTRODUCTION}

Two-beam coupling phenomena in photorefractive media ${ }^{1}$ have been used for various novel applications, such as image amplification, ${ }^{1,2}$ unidirectional ring oscillators, ${ }^{3}$ optical limiters, ${ }^{4}$ beam cleanup, ${ }^{5}$ and beam steering. ${ }^{6}$ Most of the proposed applications use steady-state energy coupling in photorefractive media with an induced index grating, which is $\pi / 2$ phase shifted from the light-interference pattern. On the other hand, the transient behavior of the energy coupling (e.g., response time) is an important factor in characterizing device performance. ${ }^{7}$ Transient energy coupling (TEC), including the interaction between two coherent beams and the induced grating formation, has been studied theoretically, and approximate analytic ${ }^{8-12}$ and numerical ${ }^{13}$ solutions have been obtained. These studies ${ }^{8-11,13}$ show that, although there is no steady-state energy exchange between the beams in unshifted dynamic holographic media, TEC occurs in such media with noninstantaneous responses. In particular, it is shown that TEC occurs in photorefractive media operating in the charge-drifting mode (which is obtained by applying an appropriate external field and using large grating periods).

In this paper we demonstrate an optical tracking filter ${ }^{14}$ based on TEC. First we qualitatively describe the physical origin of TEC by means of steady-state two-beam coupling equations. We then describe the experiment, which uses a photorefractive $\mathrm{Bi}_{12} \mathrm{SiO}_{20}$ (BSO) crystal with an external field.

\section{TRANSIENT ENERGY COUPLING}

Let us consider the following steady-state two-beam coupling equations ${ }^{15}$ [see also Fig. 1(a)]:

$$
\begin{aligned}
\frac{\mathrm{d} I_{+}}{\mathrm{d} s} & =\Gamma \frac{I_{+} I_{-}}{I_{0}}-\alpha I_{+}, \\
\frac{\mathrm{d} I}{\mathrm{~d} s} & =-\Gamma \frac{I_{+} I_{-}}{I_{0}}-\alpha I_{-}, \\
\frac{\mathrm{d}(\delta \psi)}{\mathrm{d} s} & =\Gamma^{\prime} \frac{\left(I_{+}-I_{-}\right)}{I_{0}},
\end{aligned}
$$

where $s=z / \cos \theta ; I_{0}=I_{+}+I_{-} ; \delta \psi=\psi_{+}-\psi_{-} ; I_{+}$and $I_{-}\left(\psi_{+}\right.$ and $\psi_{-}$) are the intensities (phases) of the interacting beams, respectively; $\alpha$ is the linear absorption coefficient; and $\Gamma[=2$ $\operatorname{Re}(\gamma)]$ and $\Gamma^{\prime}[=\operatorname{Im}(\gamma)]$ are the real and imaginary parts, respectively, of the (complex) two-beam coupling constant $\gamma$, which, for a degenerate case at a frequency $\omega$, is given by

$$
\gamma=\frac{\omega r_{\mathrm{eff}} n_{0}^{3}}{4 c} \frac{E_{q}\left(E_{0}+i E_{D}\right)}{E_{0}+i\left(E_{D}+E_{q}\right)}
$$

In Eq. (2) $r_{\text {eff }}$ is the relevant electro-optic coefficient, $n_{0}$ is the ordinary refractive index of the crystal, $E_{0}$ is the externally applied dc field, and $E_{D}$ and $E_{q}$ are characteristic internal electric fields of diffusion and maximum space charge, respectively. From Eq. (2), when $E_{0}=0$ or $E_{0} \gg E_{D}$ and $E_{q}, \Gamma$ is finite, and $\Gamma^{\prime}=0$. This is the case when the index grating is $\pi / 2$ phase shifted from the light-interference pattern and the steady-state energy coupling occurs. On the other hand, when the intermediate fields $E_{0}(\neq 0)$ and large grating spacings are used, there exists a region where $\left|\Gamma^{\prime}\right| \gg|\Gamma|$. This corresponds to the unshifted (local) grating case, in which there is almost no energy transfer, but phase transfer occurs between the interacting two beams provided that $I_{+} \neq I_{-}$. Because of the phase transfer, the equiphase contour of the index grating is tilted with respect to the $z$ axis in the crystal in the steady state $(t=0)$ [Fig. 1(a)]. If there is any change in the input-beam intensities, the lightinterference pattern will modify its position according to Eq. (1c). In this case; for a medium with noninstantaneous response, a transient phase mismatch between the lightinterference pattern and the index grating is created in the time period $0<t<\tau$ ( $\tau$ is the medium response time) [Fig. 1(b)]. This phase mismatch is responsible for TEC between the interacting beams. When the input-beam intensities become stationary at time $t \gg \tau$, the index grating catches up with the change of the light-interference pattern. As a result, TEC then ceases [Fig. 1(c)]. Therefore the conditions for TEC can be listed as follows:

1. The medium does not respond instantaneously, i.e., $\tau$ $\neq 0$;

2. The intensities of the two beams are different at $z=0$, i.e., $I_{+}(0) \neq I_{-}(0)$. 

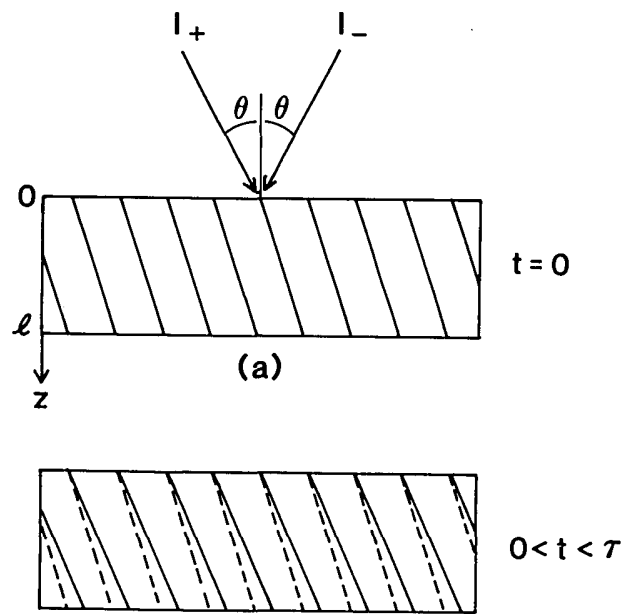

(b)

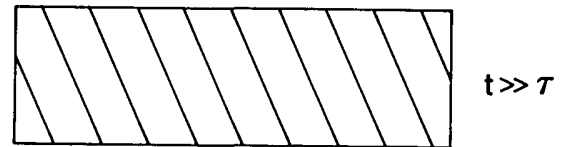

(c)

Fig. 1. Diagram to illustrate the dynamics of the holographic index grating formation. Solid lines indicate light-interference fringes, and dashed lines indicate index gratings: (a) $t=0$ (both lines are overlapped), (b) $0\langle t\langle\tau$, (c) $t \gg \tau$ (both lines are overlapped).

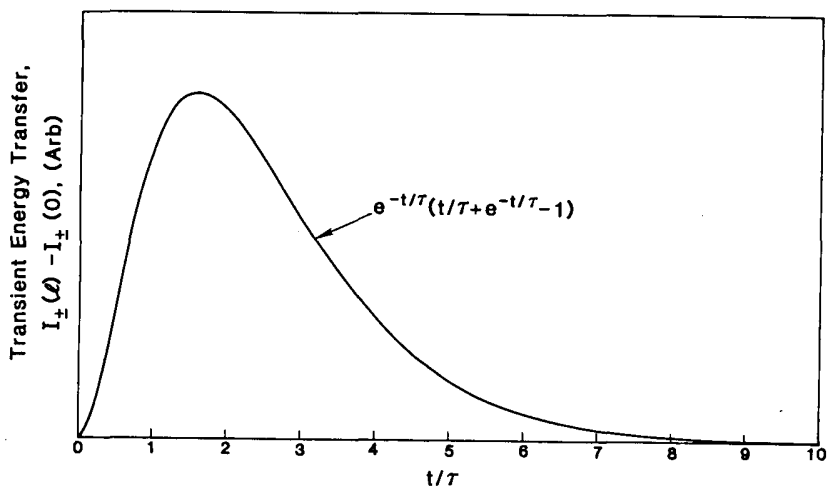

Fig. 2. Dependence of transient energy transfer on the normalized time $t / \tau$.
The analytical expression ${ }^{9,10}$ for the amount of TEC under a steplike input-beam change is given by

$$
\begin{aligned}
& I_{ \pm}(l)-I_{ \pm}(0) \propto \mp\left[I_{+}(0)-I_{-}(0)\right] I_{+}(0) I_{-}(0) \\
& \times \exp \left(-\frac{t}{\tau}\right)\left[\frac{t}{\tau}+\exp \left(-\frac{t}{\tau}\right)-1\right] .
\end{aligned}
$$

Expression (3) indicates that the energy transfer always occurs from the stronger beam to the weaker beam. A typical plot of the energy transfer versus the normalized time $t / \tau$ is shown in Fig. 2. The maximum energy transfer occurs at $t$ $\simeq 1.59 \tau$. When $\tau$ is small enough to follow a sudden change in the input-beam intensities, TEC can be approximated as the temporal differentiation of input information. In the section that follows we describe the experimental demonstration of an optical tracking filter using TEC.

\section{EXPERIMENT}

Figure 3 shows the experimental setup. A photorefractive BSO crystal $(10 \mathrm{~mm} \times 10 \mathrm{~mm} \times 3 \mathrm{~mm})$ was used. An external electric field $(6 \mathrm{kV} / \mathrm{cm})$ was applied along the crystal [001] direction to ensure that the formation of photorefractive gratings was in the charge-drifting mode. With this external field, the index modulation is high in the spatial frequency range $\Lambda^{-1}$ of $10-70 \mathrm{~mm}^{-1}$, and the phase shift between the index grating and the light-interference pattern is less than 0.1 rad. ${ }^{16,17}$ A single-longitudinal-mode argonion laser ( $\lambda=0.5145 \mu \mathrm{m}, 500-\mathrm{mW}$ output power) was expanded and divided by a beam splitter BS. The higherintensity beam $I_{-}$passed through a transparency $\mathrm{T}$ and was imaged onto the crystal (110) surface. A neutral-density filter ND was inserted into the path of the other uniform beam $I_{+}$, and the ratio $I_{-} / I_{+}$was set to approximately 10 . The angle between the two beams was chosen to be $2 \theta \simeq 1.5^{\circ}$ so that TEC was enhanced. ${ }^{10,13}$ The measurement of the two-beam coupling gain versus the input-beam polarization showed that TEC was nearly maximum when the linear polarization was nearly parallel to the crystal [001] direction. (Note that the angle of rotation due to optical activity in the crystal was $95^{\circ}$.) The beam $I_{+}$transmitted through the crystal was imaged onto a TV camera.

The $I_{-}$beam carrying the pictorial information of the

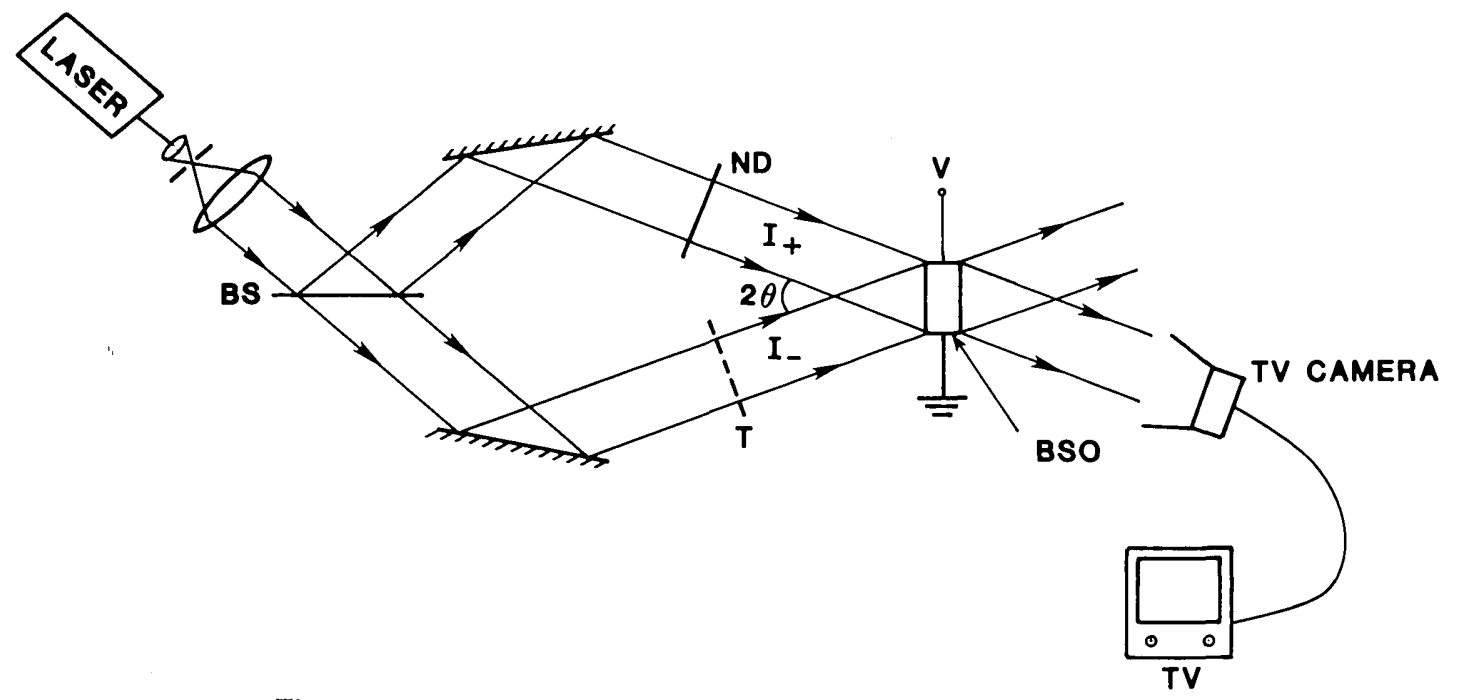

Fig. 3. Experimental setup to demonstrate the optical tracking filter. 


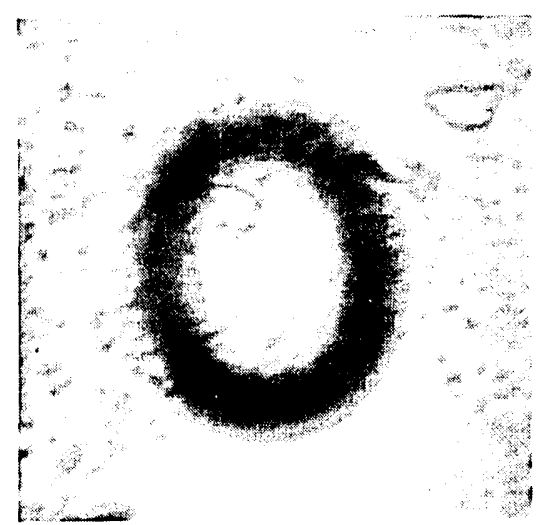

(a)

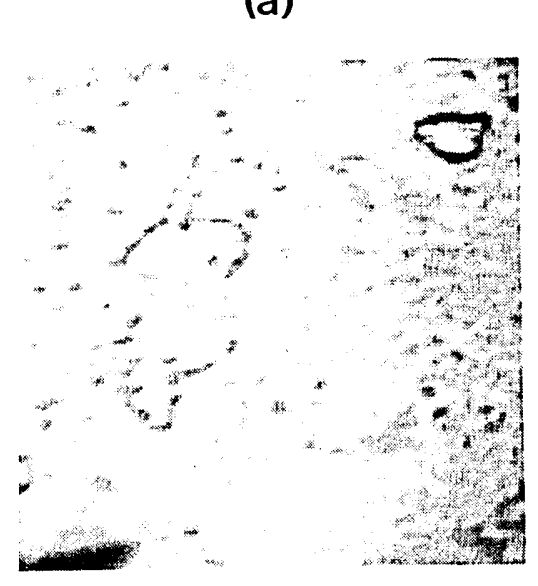

(c)

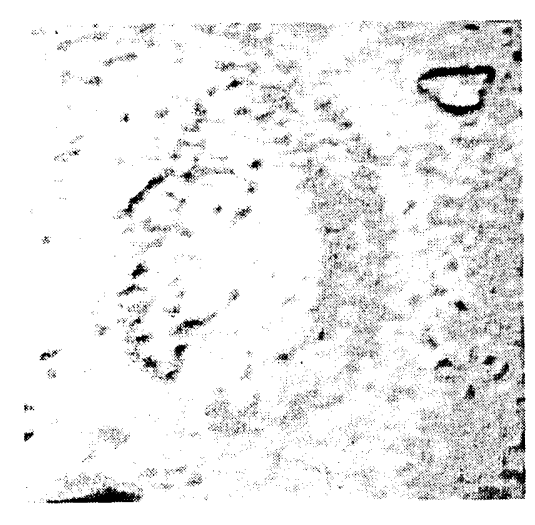

(b)

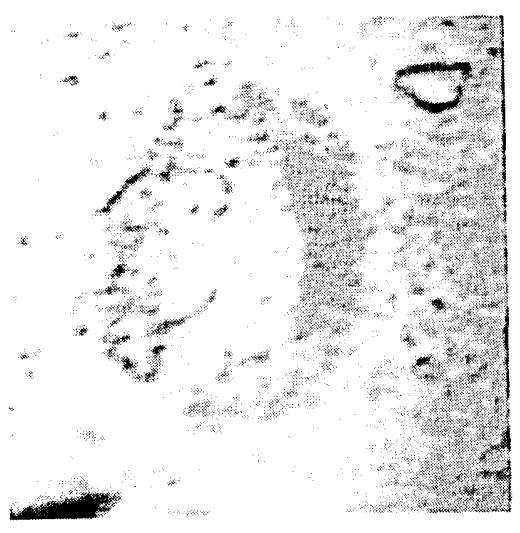

(d)

Fig. 4. (a) The $I_{-}$beam transmitted through the crystal. This beam carries the pictorial information of the letter $O$. (b) The $I_{+}$beam recorded when the transparency was moving across the beam $I_{-}$. (c) The $I_{+}$beam recorded subsequently when the transparency returned to rest. (d) The $I_{+}$beam recorded when the optical table was shaken with the transparency at rest.

letter $\mathrm{O}$ from the transparency is shown in Fig. 4(a). Some defects on the crystal surface can be seen. When the transparency is moving across the beam $I_{-}$, the corresponding letter $O$ appears on the output beam $I_{+}$[Fig. 4(b)]. Subsequently, when the transparency returns to rest, only the uniform intensity distribution of the output beam $I_{+}$is seen [Fig. 4(c)]. Figure 4(d) shows the output beam $I_{+}$when the optical table is shaken with the transparency at rest. In this case the letter $O$ is also seen. The response time in this experiment was found to be faster than $100 \mathrm{msec}$. From these figures the tracking novelty operation (in other words, the temporal differentiation) of the moving object is apparent. An optical tracking filter using a phase-conjugate interferometer together with a spatial light phase modulator was reported by Anderson et al. ${ }^{18}$ The time constant of their device was limited by the time response of the phase conjugator. Recently Cronin-Golomb et al. ${ }^{19}$ reported an optical tracking filter based on two-beam coupling in photorefractive media with nonlocal $\pi / 2$-shifted gratings. In both schemes photorefractive $\mathrm{BaTiO}_{3}$ crystals (whose response time is typically of the order of $0.1-1 \mathrm{sec}$ for an intensity of $100 \mathrm{~mW} / \mathrm{cm}^{2}$ at visible wavelengths) were used to obtain either a large phase-conjugate reflectivity or a large steady-state two-beam coupling gain for better contrast of the output images. In our case faster response materials, such as Kerr media, can be used. The contrast of the output image can be improved by increasing the intensity difference between the two input beams. The uniform background in the signal beam $I_{+}$can be reduced by using the cross-polarization coupling phenomena in the cubic crystals. ${ }^{20}$ Another possible advantage of this scheme is, as in Cronin-Golomb's scheme, that it utilizes a simple spatial light intensity modulator such as a commercially available liquid-crystal television without any modification.

\section{SUMMARY}

In this paper we have demonstrated an optical tracking filter based on TEC in a local and noninstantaneous response medium. The physical origin of TEC has also been illustrated. This scheme is comparatively simple and has the potential to use faster-response materials.

\section{ACKNOWLEDGMENTS}

The portion of this research performed at the California Institute of Technology was supported by the U.S. Air Force Office of Scientific Research and by the U.S. Army Research Office, Durham, North Carolina. 


\section{REFERENCES}

1. N. V. Kukhtarev, V. B. Markov, S. G. Odulov, M. S. Soskin, and V. L. Vinetskii, Ferroelectrics 22, 949, 961 (1979).

2. T. Tschudi, A. Herden, J. Goltz, H. Klumb, F. Laeri, and F. Albers, IEEE J. Quantum Electron. QE-19, 1493 (1986).

3. J. O. White, M. Cronin-Golomb, B. Fisher, and A. Yariv, Appl. Phys. Lett. 40, 450 (1982).

4. M. Cronin-Golomb and A. Yariv, J. Appl. Phys. 57, 4906 (1985).

5. A. E. Chiou and P. Yeh, Opt. Lett. 10,621 (1985); S.-K. Kwong and A. Yariv, Appl. Phys. Lett. 48, 564 (1986).

6. D. Rak, I. Ledonx, and J. P. Huingnard, Opt. Commun. 49, 302 (1984).

7. G. C. Valley and M. B. Klein, Opt. Eng. 22, 704 (1983).

8. V. L. Vinetskii and N. V. Kukhtarev, Sov. Tech. Phys. Lett. 2, 364 (1976).

9. V. L. Vinetskii, N. V. Kukhtarev, and M. S. Soskin, Sov. J. Quantum Electron. 7, 230 (1977).

10. N. Kukhtarev, V. Markov, and S. Odulov, Opt. Commun. 23, 338 (1977).
11. L. Solymar and J. M. Heaton, Opt. Commun. 51, 76 (1984).

12. M. Cronin-Golomb, in Digest of Topical Meeting on Photorefractive Materials, Effects, and Devices (Optical Society of America, Washington, D.C., 1987), paper ThC5.

13. J. M. Heaton and L. Solymar, Opt. Acta 32, 397 (1985).

14. The main part of this work is from N. S.-K. Kwong, in Digest of Topical Meeting on Photorefractive Materials, Effects, and Devices (Optical Society of America, Washington, D.C., 1987), paper ThC7.

15. S.-K. Kwong, M. Cronin-Golomb, and A. Yariv, IEEE J. Quantum Electron. QE-22, 1508 (1986).

16. M. Peltier and F. Micheron, J. Appl. Phys. 48, 3683 (1977).

17. J. P. Huignard and A. Marrakchi, Opt. Commun. 38, 249 (1981).

18. D. Z. Anderson, D. M. Linger, and J. Feinberg, Opt. Lett. 12,123 (1987).

19. M. Cronin-Golomb, A. M. Biernacki, C. Lin, and H. Kong, Opt. Lett. 12, 1029 (1987).

20. A. Partovi, E. M. Garmire, and L. J. Cheng, Appl. Phys. Lett. 51, 299 (1987); P. Yeh, J. Opt. Soc. Am. B 4, 1382 (1987).

\section{Norman Sze-Keung Kwong}

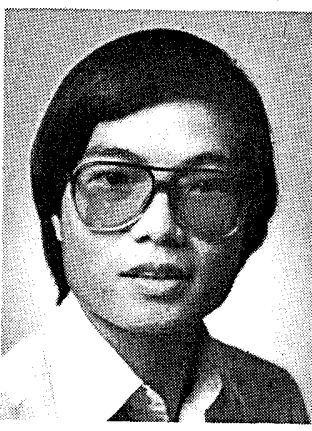

Norman Sze-Keung Kwong was born in Hong Kong on August 5, 1958. He received the B.Sc. degree in physics from the University of Hong Kong in 1980, the M.S. degree in physics from the University of Southern California, Los Angeles, California, in 1981, and the Ph.D. degree in physics from the California Institute of Technology (Caltech), Pasadena, California, in 1986. As an undergraduate, he conducted experiments to measure cosmic-ray muon intensity. From 1981 to 1986 he was at Caltech studying twowave and four-wave mixing, phase conjugation, image processing, associative memories, and bistabilities with photorefractive materials. Since 1986 he has been a staff scientist at Ortel Corporation. $\mathrm{He}$ continues his research on imaging processing and optical-fiber sensors using photorefractive materials. He manages a program to develop high-power GaAlAs and InGaAsP superluminescent diodes.

\section{Yasuo Tamita}

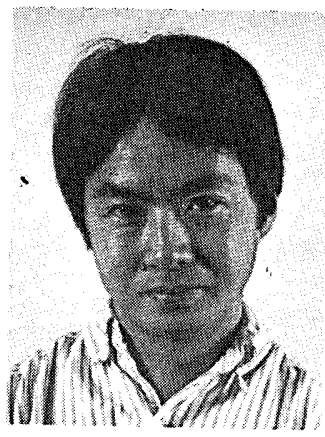

Yasuo Tamita was born in Sapporo, Japan, on September 28, 1955 . He received the B.E. and M.E. degrees, both in electronic engineering, from Hokkaido University, Sapporo, in 1978 and 1980, respectively, and the M.S. degree in electrical engineering from the California Institute of Technology in 1985. During his graduate study at the Research Institute of Applied Electricity, Hokkaido University, he investigated the statistical properties of white light and polychromatic speckles. In 1980 he joined the Canon Research Center, Tokyo, where he worked on magneto-optics and its application to erasable optical memories.
Since 1984 he has been with Caltech on leave from the Canon Research Center. He is now a Ph.D. candidate in electrical engineering at Caltech. His current research interests include propagation characteristics of phase-conjugate waves in strongly scattering media and their applications, photorefractive nonlinear optics, nonlinear-optical phenomena in passive and active waveguides, and optical computing. He has published many papers in the areas of speckles, magneto-optics, and phase conjugation. He is a member of the Japan Society of Applied Physics and the Optical Society of America.

\section{Amnon Yariv}

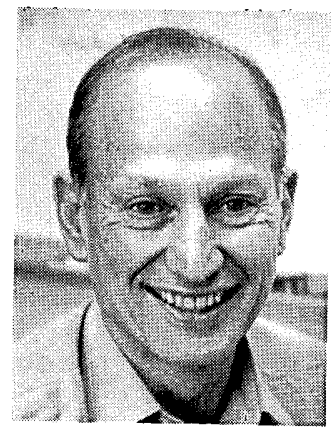

Amnon Yariv, a native of Israel, obtained the B.S. (1954), M.S. (1956), and Ph.D. (1958) degrees in electrical engineering from the University of California at Berkeley. He joined the Bell Telephone Laboratories, Murray Hill, New Jersey, in 1959, joining the early stages of the laser effort. He came to the California Institute of Technology in 1964 as an associate professor of electrical engineering, becoming a professor in 1966 . In 1980 he became the Thomas G. Myers Professor of Electrical Engineering and Applied Physics. On the technical side, he took part (with various co-workers) in the discovery of a number of early solid-state laser systems, in proposing and demonstrating semiconductor-based integrated optics technology, and in pioneering the field of phaseconjugate optics. His present research efforts are in the areas of nonlinear optics, semiconductor lasers, and integrated optics. $\mathrm{He}$ has published widely in the laser and optics fields (approximately 300 papers) and has written a number of basic texts in quantum electronics, optics, and quantum mechanics. Dr. Yariv is a member of the American Physical Society, Phi Beta Kappa, the American Academy of Arts and Sciences, and the National Academy of Engineering and is a Fellow of the Institute of Electrical and Electronics Engineers (IEEE) and the Optical Society of America. He has received the 1980 Quantum Electronics Award of the IEEE, the 1985 University of Pennsylvania Pender Award, and the 1986 Optical Society of America Ives Medal. Dr. Yariv is a founder and chairman of the board of Ortel Corporation. 